\title{
Trefftz Discontinuous Galerkin basis functions for a class of Friedrichs systems coming from linear transport
}

\author{
Christophe Buet ${ }^{1,5}$, Bruno Despres ${ }^{2,3,5}$, Guillaume Morel $^{4,5}$ \\ ${ }^{1}$ CEA, DAM, DIF, F-91297 Arpajon, France \\ 2 Laboratoire Jacques-Louis Lions, Sorbonne Université, F-75005 Paris, France \\ ${ }^{3}$ Institut Universitaire de France \\ ${ }^{4}$ Inria Rennes, Campus de Beaulieu, 263 Avenue Général Leclerc, F-35042 Rennes, France.
}

\begin{abstract}
The Trefftz discontinuous Galerkin (TDG) method provides natural well-balanced (WB) and asymptotic preserving (AP) discretization since exact solutions are used locally in the basis functions. However, one difficult point may be the construction of such solutions which is a necessary first step in order to apply the TDG scheme. This works deals with the construction of solutions to Friedrichs systems with relaxation with application to the spherical harmonics approximation of the transport equation (the so-called $P_{N}$ models). Various exponential and polynomial solutions are constructed. Two numerical tests on the $P_{3}$ model illustrate the good accuracy of the TDG method. They show that the exponential solutions lead to accurate schemes to capture boundary layers on a coarse mesh and that a combination of exponential and polynomial solutions is efficient in a regime with vanishing absorption coefficient.
\end{abstract}

\section{Introduction}

Trefftz methods use exact solutions of a given partial differential equation (PDEs) as basis functions for the numerical approximation. Nevertheless, despite their great potential for the improvement of Finite Element Methods (FEM) solvers [34] or for the enrichment of Discontinuous Galerkin (DG) approximations [33], Trefftz methods are scarcely used nowadays [24]. One reason is the limited number of different Trefftz basis functions available in the literature. Indeed, for Trefftz methods, one has to construct new basis functions for every different class of PDEs: only after this task is accomplished, then it is possible to measure the gain in accuracy with respect to more conventional methods, or to incorporate them in general FEM or DG solvers $[9,11,17,23,29]$. One of the difficulty when applying the TDG method is therefore to find solutions to the system considered. Our objective in this work is precisely to contribute to this global program by constructing new exact solutions to general Friedrichs systems with relaxation, in view of their use as basis functions for an original Trefftz Discontinuous Galerkin (TDG) method. The Friedrichs systems with have in mind can be seen as extensions of wave like models already treated in $[18,29]$. With respect to the literature on Trefftz methods, we introduce a new generic non-zero relaxation matrix which is representative of dissipation mechanisms often encountered in complex physics.

More specifically, the model problem throughout this work writes as

$$
\left(A_{0} \partial_{t}+A_{1} \partial_{x}+A_{2} \partial_{y}\right) \mathbf{u}(t, \mathbf{x})=-R(\mathbf{x}) \mathbf{u}(t, \mathbf{x}),
$$

where the space coordinates is $\mathbf{x}=(x, y)$, the real matrices are symmetric $A_{1}=A_{1}^{T}, A_{2}=A_{2}^{T}$, $R(\mathbf{x})=R(\mathbf{x})^{T} \in \mathbb{R}^{m \times m}$ is piecewise constant per cell and the vectorial unknown is $\mathbf{u} \in \mathbb{R}^{m}$. The

\footnotetext{
${ }^{5}$ E-mail addresses: christophe.buet@cea.fr, despres@ann.jussieu.fr, guillaume.morel@inria.fr
} 
matrix on the right hand side may strongly depend on the space variable $\mathbf{x}$. This model can be motivated as an angular discretization of a linear Boltzmann equation which is a transport/kinetic equation where the physics is modeled with linear interaction relaxation operators (4). We adopt a convention, which comes from the physics of transfer or neutron propagation [22], that the matrices $A_{1}$ and $A_{2}$ have a block structure

$$
A_{1}=c\left(\begin{array}{cc}
0 & A \\
A^{T} & 0
\end{array}\right) \in \mathbb{R}^{m \times m}, \quad A_{2}=c\left(\begin{array}{cc}
0 & B \\
B^{T} & 0
\end{array}\right) \in \mathbb{R}^{m \times m},
$$

where $A, B \in \mathbb{R}^{m_{e} \times m_{o}}$ are constant rectangular matrices $\left(m_{e}+m_{o}=m\right)$. The coefficient $c>0$ is a constant non dimensional wave velocity. For the purposes of mathematical manipulation, the first matrix is $A_{0}=\varepsilon I_{m} \in \mathbb{R}^{m \times m}$ where $I_{m} \in \mathbb{R}^{m \times m}$ is the identity matrix and $0<\varepsilon \leq 1$ indicates a possible rescaling of the time variable. If the relaxation matrix on the right hand side is zero, then our model is the same as in $[18,29,15]$. The originality of our methods comes from the non zero relaxation matrix. A natural structure which models relaxation mechanisms is $R+R^{t} \geq 0$. In our work, we follow closely the convention proposed in [22] by taking

$$
R=\left(\begin{array}{cc}
R_{1} & 0 \\
0 & R_{2}
\end{array}\right) \in \mathbb{R}^{m \times m},
$$

where $R_{1}$ and $R_{2}$ are both diagonal matrices $R_{1}:=\operatorname{diag}\left(\varepsilon \sigma_{a}, \sigma_{t}, \ldots, \sigma_{t}\right) \in \mathbb{R}^{m_{e} \times m_{e}}, R_{2}:=\sigma_{t} I_{m_{o}} \in$ $\mathbb{R}^{m_{o} \times m_{o}}$, with $I_{m_{o}}$ the identity matrix of $\mathbb{R}^{m_{o} \times m_{o}}$. For transfer models [22] $\sigma_{a} \geq 0$ is the absorption coefficient and $\sigma_{s} \geq 0$ is the scattering coefficient. The weighted sum of the scattering and absorption coefficients will be denoted as

$$
\sigma_{t}:=\sigma_{t}^{\varepsilon}:=\varepsilon \sigma_{a}+\frac{\sigma_{s}}{\varepsilon}, \quad \sigma_{a}, \sigma_{s} \in \mathbb{R}_{+} .
$$

When the scaling parameter $\varepsilon \rightarrow 0$, the model problem admits a diffusion limit $[22,8]$. General references which provide accurate numerical methods for the diffusion limit are $[3,8,19,27]$ for asymptotic-preserving methods and $[13,20,21,26]$ for well-balanced methods. In principle, Trefftz method may be very efficient in the diffusion limit since the exact solutions in the cell have a perfect balance between the transport terms (matrices $A_{1}$ and $A_{2}$ ) and the relaxation (matrix $R$ ). A simple proof that the Trefftz scheme is indeed Asymptotic Diffusion Preserving can be found in [31].

The relaxation matrix $R(\mathbf{x})$ can be discontinuous as well in applications. This is typical of the physics of transfer at the interface between two different materials and of neutron propagation: in the application illustrated at the end of this work, the unknown $\mathbf{u}$ comes from an angular discretization of a populations of neutrons and relaxation coefficients model the interaction of neutrons with matter; the issue is that this matter is different on both sides of an interface. Boundary layers may occur when $\sigma_{a}, \sigma_{s}$ vary significantly and that the transport equation tends to a diffusion limit when $\sigma_{s}$ is high. These two phenomena are challenging for numerical methods and the research is active in the scientific community. The literature is scarce on numerical methods for boundary layers. It has been highlighted in [31] that TDG method naturally leads to schemes adapted to such problems.

Some aspects of the constructive methods developed hereafter are strongly related to the particular relaxation matrix (3), however the general strategy can be adapted to different relaxation matrices.

This paper is organized as follows. In Section 2, the Friedrichs system with relaxation is constructed as the angular discretization of a kinetic equation: in the literature it is called the $P_{N}$ model and its main properties are given; these properties are directly connected to invariance principles which are common to many different models. In Section 3, we determine Trefftz families of exponential exponential and polynomial solutions to our model problem. An interesting property is that some of the exponential solutions degenerate when $\sigma_{a} \rightarrow 0$, meaning that they become linear dependent for $\sigma_{a}=0$. The relation between the degenerative exponentials and the polynomials is studied with the Birkhoff/Abu-Shumays method which is based on an exact property of the underlying kinetic equation (4). Next, in Section 4, the stationary solutions (polynomials and exponentials) are explicitly 
calculated for the $P_{1}$ and $P_{3}$ models. In the final Section, a numerical example with boundary layers is provided for the $P_{3}$ model in Section 5: in terms of accuracy, it shows an important improvement with respect to more standard DG methods. Another test problem illustrates the advantage of the method for a test problem in a diffusion regime. These numerical examples illustrate the fundamental interest of using TDG methods whence classical polynomial based numerical methods have low accuracy for a given problem.

\section{Principles of a $P_{N}$ model and some properties}

$P_{N}$ models have their own physical interest for the angular modeling of kinetic equations. In the context of this work, they also have the virtue that it makes possible to construct a system like (1) of arbitrary size, and to explain the structure in relation with invariance principles. In what follows we restrict the presentation to the most fundamental aspects and provide two basic examples which are the $P_{1}$ model and the $P_{3}$ model.

Let us consider the spherical approximation of the transport equation which reads

$$
\partial_{t} f(t, \mathbf{x}, \boldsymbol{\Omega})+\boldsymbol{\Omega} \cdot \nabla f(t, \mathbf{x}, \boldsymbol{\Omega})=-\sigma_{a}(\mathbf{x}) f(t, \mathbf{x}, \boldsymbol{\Omega})+\sigma_{s}(\mathbf{x})(|f|-f(t, \mathbf{x}, \boldsymbol{\Omega})),
$$

where $f$ is the distribution function, $t$ the time variable, $\mathbf{x} \in \mathbb{R}^{d}$ the space variable, $\boldsymbol{\Omega}$ the direction and $|f|=\frac{1}{4 \pi} \int_{S^{2}} f\left(t, \mathbf{x}, \boldsymbol{\Omega}^{\prime}\right) d \boldsymbol{\Omega}^{\prime}$ is the mean of $f$. The absorption and scattering coefficients model the interaction with the matter. Start from the spherical harmonics written for simplicity as $\left(\varphi_{n}(\boldsymbol{\Omega})\right)_{n \geq 1}$ with the orthonormal relation $\left\langle\varphi_{n}(\boldsymbol{\Omega}), \varphi_{n^{\prime}}(\boldsymbol{\Omega})\right\rangle=\delta_{n, n^{\prime}}$ The idea is to plug a truncated representation

$$
f^{m}(t, \mathbf{x}, \boldsymbol{\Omega})=\sum_{n=1}^{m} u_{n}(t, \mathbf{x}) \varphi_{n}(\boldsymbol{\Omega})
$$

in (4) and to consider the moment system

$$
\begin{gathered}
\left\langle\partial_{t} f^{m}(t, \mathbf{x}, \boldsymbol{\Omega}), \varphi_{n}(\boldsymbol{\Omega})\right\rangle+\left\langle\boldsymbol{\Omega} \cdot \nabla f^{m}(t, \mathbf{x}, \boldsymbol{\Omega}), \varphi_{n}(\boldsymbol{\Omega})\right\rangle \\
=-\sigma_{a}(\mathbf{x})\left\langle f^{m}(t, \mathbf{x}, \boldsymbol{\Omega}), \varphi_{n}(\boldsymbol{\Omega})\right\rangle+\sigma_{s}(\mathbf{x})\left\langle\left|f^{m}\right|, \varphi_{n}(\boldsymbol{\Omega})\right\rangle-\sigma_{s}(\mathbf{x})\left\langle f^{m}, \varphi_{n}(\boldsymbol{\Omega})\right\rangle, \quad 1 \leq n \leq m .
\end{gathered}
$$

One obtains a system like (1) but in dimension 3. The unknown is $u=\left(u_{1}, \ldots, u_{m}\right)$. The total size of the system is the sum of the number of even moments (related to the spherical harmonics) and the number of odd moments (related to the spherical harmonics), that is $m=m_{e}+m_{o}$. Using the more natural notation $\varphi_{n}=Y_{l, m}$ for spherical harmonics (the highest degree is $N \geq l \geq 0$ ), one has $m=\frac{1}{2}(N+1)(N+2), m_{e}=\frac{1}{4}(N+1)^{2}, m_{o}=\frac{1}{4}(N+1)(N+3)$. This is the reason of the denomination $P_{N}$ model. Additional properties of $P_{N}$ models can be noted. For convenience we briefly recall what can be found in the references [16, 22, 32]. These properties are deeply related to invariance principles. a) In practice, the $P_{N}$ model is rarely applied for even values of $N$ (see for example [16, Section 2] for a discussion on the benefits of considering $N$ odd) and it is therefore natural to consider only the case $N$ odd in our analysis.

b) In [22], it is proved that the decoupling between even moments and odd moments yields a block structure like (2).

c) Provided $\sigma_{a}$ and $\sigma_{s}$ are invariant with respect to $z$, then the system is invariant by translation with respect to $z$. So it is possible to make the assumption that the solution does not depends on the variable $z$ that is $\partial_{z} \mathbf{u}=0$.

d) It is possible to assume that the solution has a mirror symmetry with respect to the plan $x y$. In three dimensions, it can be interpreted as pure reflective conditions at the top and bottom boundaries of the domain, as illustrated Figure 1. This assumption equates some moments, so reduces the size of the system.

e) Finally, a $P_{N}$ model has to satisfy some rotational invariance principles. With the assumptions c) and d) this rotational invariance is expressed in the plan $x y$. The plan $x z$ may also be a possible 


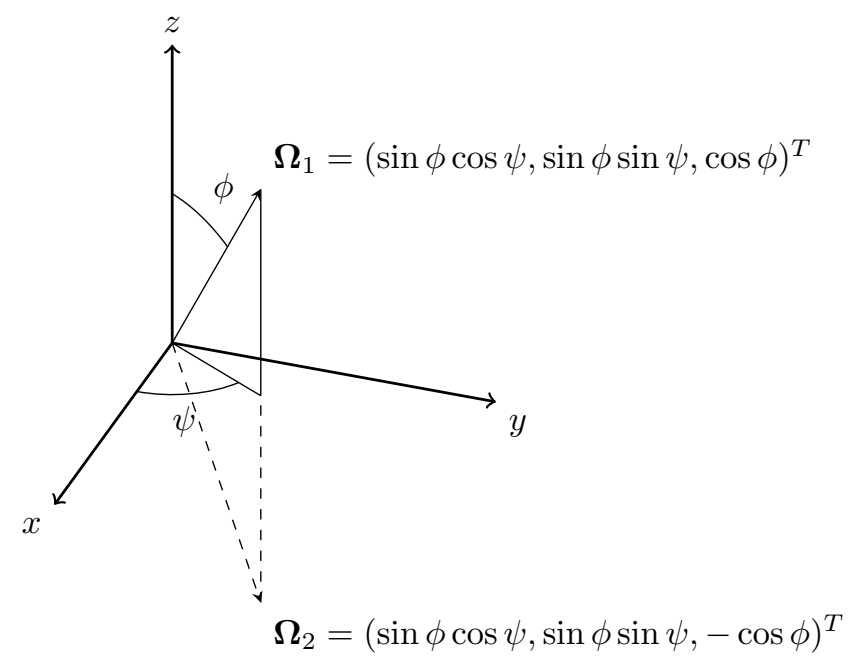

Figure 1: Representation of directions $\boldsymbol{\Omega}_{1}$ and $\boldsymbol{\Omega}_{2}$. If $\mathbf{u}$ is an even function of $\cos \phi$ then $\mathbf{u}\left(t, \mathbf{x}, \boldsymbol{\Omega}_{1}\right)=$ $\mathbf{u}\left(t, \mathbf{x}, \boldsymbol{\Omega}_{2}\right)$.

choice $[7,8]$, however the rotation matrix associated with the spherical harmonics is more difficult to calculate $[6,25,32]$. The matrix representations of the rotation operators in the basis of spherical harmonics is needed $[6,12,32]$

$$
\mathcal{U}(\alpha, \beta, \gamma) \in \mathbb{R}^{m^{3 D} \times m^{3 D}},
$$

where $\alpha, \beta$ and $\gamma$ denotes rotation around the axes $O_{z}, O_{y}$ and $O_{z}$ respectively. To simplify the matrix $\mathcal{U}$ we consider a rotation $\theta$ in the plan $x y$ only and denote

$$
\mathcal{U}_{\theta}:=\mathcal{U}(0,0, \theta) \in \mathbb{R}^{m^{3 D} \times m^{3 D}} .
$$

Let $\mathbf{y}(\boldsymbol{\Omega})$ denotes the vector made of the spherical harmonics arranged in a particular order, see [30, Section 4-1.1]. Then the matrix $\mathcal{U}$ represents the orthogonal transformations on $\mathbf{y}(\boldsymbol{\Omega})$. That is for an orthogonal matrix $Q \in \mathbb{R}^{3 \times 3}$ one has

$$
\mathbf{y}(Q \boldsymbol{\Omega})=\mathcal{U}(\alpha, \beta, \gamma) \mathbf{y}(\boldsymbol{\Omega}),
$$

where $\alpha, \beta$ and $\gamma$ are the angles of the rotation associated with the matrix $Q$ in $\mathbb{R}^{3}$. For an explicit formula of the matrix $\mathcal{U}_{\theta}$, see [30, Section 4.1.3.2].

Proposition 2.1. Assume a) to e). Then the $P_{N}$ model written as (1) is invariant under rotation. More precisely, if $\mathbf{u}(t, x, y)$ is solution to (1) then the function $U_{\theta} \mathbf{u}(t, x \cos \theta+y \sin \theta,-x \sin \theta+y \cos \theta)$, $\theta \in[0,2 \pi)$, is also solution to (1).

We can now give some properties which can be proved for a general $P_{N}$ model satisfying a) to e). The verification is more tedious, this is why we just admit these properties without detailing the proofs which however can be found in [30, Section 4.1]. However it is straightforward to verify all these properties on 2 examples which are explicitly given at the end of this section. The first one is the $P_{1}$ model $(m=3)$, refer to Subsection 2.1. The second example is the $P_{3}$ model $(m=10)$, refer to Subsection 2.2.

Proposition 2.2 (Invertibility of $A A^{T}$ and $B B^{T}$ ). The symmetric matrix $A A^{T}$ is invertible and all its eigenvalues are strictly positive. 
Proposition 2.3 (Eigenvalues of $\left.\left(A A^{T}\right)^{-1} R_{1}\right)$. The eigenvalues $\mu_{i}$ of the matrix $\left(A A^{T}\right)^{-1} R_{1}$ are strictly positive when $\sigma_{a}>0$ and non negative when $\sigma_{a}=0$.

An important property will be the degeneracy of the lowest eigenvalue as $\sigma_{a} \rightarrow 0$.

Proposition 2.4. Assume $\sigma_{s}>0$. The lowest eigenvalue of $\left(A A^{T}\right)^{-1} R_{1}$ is such that $\mu_{1} \rightarrow 0$ as $\sigma_{a} \rightarrow 0$.

Finally, one can count the number of distinct couples of eigenvalue/eigenvector of the matrix $\left(A A^{T}\right)^{-1} R_{1}$. This gives the total number of stationary exponential solutions in 1D (see the proof of Theorem 3.1).

Proposition 2.5. The eigenvectors of $\left(A A^{T}\right)^{-1} R_{1} \in \mathbb{R}^{m_{e} \times m_{e}}$ form a basis of $\mathbb{R}^{m_{e}}$.

Next we give the $P_{1}$ model which is the simplest example which satisfies all these properties. The matrix $A$ reads $A=(1,0)$ and of course the matrix $A A^{T}=1$ is invertible.

\subsection{The $P_{1}$ model}

For the $P_{1}$ model $m=3, m_{e}=1, m_{o}=2$ and the matrices $A_{1}, A_{2}, R$ and $U_{\theta}$ are

$$
\begin{aligned}
A_{1} & =\frac{1}{\sqrt{3}}\left(\begin{array}{ccc}
0 & 1 & 0 \\
1 & 0 & 0 \\
0 & 0 & 0
\end{array}\right), & A_{2} & =\frac{1}{\sqrt{3}}\left(\begin{array}{ccc}
0 & 0 & 1 \\
0 & 0 & 0 \\
1 & 0 & 0
\end{array}\right), \\
R & =\left(\begin{array}{ccc}
\sigma_{a} & 0 & 0 \\
0 & \sigma_{t} & 0 \\
0 & 0 & \sigma_{t}
\end{array}\right), & U_{\theta} & =\left(\begin{array}{ccc}
1 & 0 & 0 \\
0 & \cos \theta & -\sin \theta \\
0 & \sin \theta & \cos \theta
\end{array}\right) .
\end{aligned}
$$

The straightforward extension in 3D can be found in [30][page 49].

\subsection{The $P_{3}$ model}

For the $P_{3}$ model one has $m=10, m_{e}=4, m_{o}=6$ and the matrices read [22]

$$
\begin{gathered}
A=\left(\begin{array}{cccccc}
0 & \frac{1}{\sqrt{3}} & 0 & 0 & 0 & 0 \\
\frac{1}{\sqrt{5}} & 0 & \sqrt{\frac{3}{14}} & -\frac{1}{\sqrt{70}} & 0 & 0 \\
0 & -\frac{1}{\sqrt{15}} & 0 & 0 & \sqrt{\frac{6}{35}} & 0 \\
0 & \frac{1}{\sqrt{5}} & 0 & 0 & -\frac{1}{\sqrt{70}} & \sqrt{\frac{3}{14}}
\end{array}\right), \quad B=\left(\begin{array}{cccccc}
\frac{1}{\sqrt{3}} & 0 & 0 & 0 & 0 & 0 \\
0 & \frac{1}{\sqrt{5}} & 0 & 0 & -\frac{1}{\sqrt{70}} & -\sqrt{\frac{3}{14}} \\
-\frac{1}{\sqrt{15}} & 0 & 0 & \sqrt{\frac{6}{35}} & 0 & 0 \\
-\frac{1}{\sqrt{5}} & 0 & \sqrt{\frac{3}{14}} & \frac{1}{\sqrt{70}} & 0 & 0
\end{array}\right), \\
R_{1}=\left(\begin{array}{cccc}
\varepsilon \sigma_{a} & 0 & 0 & 0 \\
0 & \sigma_{t} & 0 & 0 \\
0 & 0 & \sigma_{t} & 0 \\
0 & 0 & 0 & \sigma_{t}
\end{array}\right), R_{2}=\sigma_{t} I_{m_{o}},
\end{gathered}
$$

where $I_{m_{o}}$ is the identity matrix of $\mathbb{R}^{m_{o} \times m_{o}}$. The matrices $A_{1}$ and $A_{2}$ are given by (2). The rotation matrix is provided in [30][formula (4.20) page 53]. Even if tedious, it is elementary to check that all the properties hold.

\section{Construction of local exact solutions}

In order to develop a TDG method for (1), one needs to construct good basis functions which are exact solutions to the system. For the kind of problem we have in mind, it is always possible to assume that 
all matrices are piecewise constants. We propose 3 different methods to construct stationary basis functions.

1) The first method construct exponential functions. It has strong reminiscence to case solutions [10] to the transport equation $[19,4,5]$. The Theorem 3.1 shows how to construct exponential solutions when $\sigma_{a} \geq 0$ which can be used as basis functions. This is based on the eigenvalues and eigenvectors of a particular spectral problem.

2) The second method is more a complement on the first one. It is based on the secular equation which gives information about the eigenvalues of the spectral problem.

3) The third one shows how the Birkhoff and Abu-Shumays approach can be used to deduce polynomial solutions of a general $P_{N}$ model. An important restriction is vanishing absorption $\sigma_{a}=0$.

A 4th one generalizes the construction to non stationary solutions. It is postponed in the appendix since it is somehow a simple extension.

\subsection{Exponential solutions}

Take a constant vector $\mathbf{d}=(\cos \theta, \sin \theta)$ for a given angle $0 \leq \theta<2 \pi$. The general idea is to plug the Ansatz

$$
\mathbf{u}(\mathbf{x})=\mathbf{r} e^{\lambda \mathbf{d}^{T} \mathbf{x}}, \quad \mathbf{r} \neq 0
$$

in the stationary equation (1). One gets the spectral problem

$$
\lambda\left(\cos \theta A_{1}+\sin \theta A_{2}\right) \mathbf{r}=-R \mathbf{r} .
$$

That is $\lambda$ is seen as an eigenvalue of the matrix $-R$ in the metric associated to the matrix $M=$ $\cos \theta A_{1}+\sin \theta A_{2}$. Both matrices are real symmetric, however $M$ is highly degenerated because in general it has positive, negative and vanishing eigenvalues. Indeed it is already the case for $A_{1}$ for the $P_{1}$ model. However it is possible to construct the general solutions by carefully studying the structure (2) and (3) of the matrices.

Theorem 3.1. Consider the system (1-3) with the result of Propositions 2.1 and 2.2. Let $\sigma_{t}>0$ and $\mathbf{w}_{1}, \ldots, \mathbf{w}_{m_{e}} \in \mathbb{R}^{m_{e}}$ be the eigenvectors of the matrix $\left(A A^{T}\right)^{-1} R_{1}$ associated with the eigenvalues $\mu_{1}, \ldots, \mu_{m_{e}}$. Let $\boldsymbol{\chi}_{i}=-\sqrt{\frac{\mu_{i}}{\sigma_{t}}} A^{T} \mathbf{w}_{i} \in \mathbb{R}^{m_{o}}, \mathbf{z}_{i}=\left(\mathbf{w}_{i}^{T}, \boldsymbol{\chi}_{i}^{T}\right)^{T} \in \mathbb{R}^{m}$ and $\mathbf{d}_{k}=\left(\cos \theta_{k}, \sin \theta_{k}\right)^{T} \in \mathbb{R}^{2}$. Let $U_{\theta_{k}}$ be the rotation matrix (6). Then the exponential functions

$$
\left(\mathbf{v}_{i}\right)_{k}(\mathbf{x})=U_{\theta_{k}} \mathbf{z}_{i} e^{\frac{1}{c} \sqrt{\sigma_{t} \mu_{i}} \mathbf{d}_{k}^{T} \mathbf{x}}, \quad i=1, \ldots, m_{e},
$$

are stationary solutions to the system.

Proof. We search for solutions under the form (9), that is with slightly different notations

$$
\widetilde{\mathbf{v}}(\mathbf{x})=\widetilde{\mathbf{z}} e^{\lambda \mathbf{d}_{k}^{T} \mathbf{x}} \in \mathbb{R}^{m}
$$

with $\mathbf{d}_{k}=\left(\cos \theta_{k}, \sin \theta_{k}\right)^{T}, \lambda \in \mathbb{R}$ and $\widetilde{\mathbf{z}} \in \mathbb{R}^{m}$. Using Proposition 2.1 the function $\mathbf{v}(x, y)=$ $U_{-\theta_{k}} \widetilde{\mathbf{v}}\left(x \cos \theta_{k}-y \sin \theta_{k}, x \sin \theta_{k}+y \cos \theta_{k}\right)$ is also a solution to the $P_{N}$ model. It can be written under the form

$$
\mathbf{v}(x, y)=\mathbf{z} e^{\lambda x} \in \mathbb{R}^{m}
$$

$\mathbf{z} \in \mathbb{R}^{m}$. Inserting (12) in (1) gives after removing the exponentials $\lambda A_{1} \mathbf{z}=-R \mathbf{z}$. We use the decomposition $\mathbf{z}=\left(\mathbf{w}^{T}, \boldsymbol{\chi}^{T}\right)^{T} \in \mathbb{R}^{m}$ where $\mathbf{w} \in \mathbb{R}^{m_{e}}$ and $\boldsymbol{\chi} \in \mathbb{R}^{m_{o}}$. Using the particular form of the matrix $A_{1}$ and $R$, one has

$$
\left\{\begin{array}{l}
\lambda c A \chi=-R_{1} \mathbf{w} \\
\lambda c A^{T} \mathbf{w}=-R_{2} \chi
\end{array}\right.
$$


Multiplying the second equation by $\lambda c A$ and then using the first equation on the right hand side with $R_{2}^{-1}=\frac{1}{\sigma_{t}} I_{m_{o}}$ gives $\lambda^{2} c^{2} A A^{T} \mathbf{w}=\sigma_{t} R_{1} \mathbf{w}$. From Proposition 2.2 the matrix $A A^{T}$ is invertible therefore one obtains the reduced spectral equation

$$
\left(A A^{T}\right)^{-1} R_{1} \mathbf{w}=\frac{\lambda^{2} c^{2}}{\sigma_{t}} \mathbf{w} .
$$

If $\mathbf{w} \in \mathbb{R}^{m_{e}}$ is an eigenvector of the matrix $\left(A A^{T}\right)^{-1} R_{1}$ associated with the eigenvalue $\mu(\mu \geq 0$ from Proposition 2.3), one can take $\lambda= \pm \sqrt{\sigma_{t} \mu} / c$. First we consider the case $\lambda=\sqrt{\sigma_{t} \mu} / c$, the case $\lambda=-\sqrt{\sigma_{t} \mu} / c$ will be discuss later. The second equation in (13) gives $\chi=-\sqrt{\mu / \sigma_{t}} A^{T} \mathbf{w} \in \mathbb{R}^{m_{o}}$. One concludes that the one dimensional function $\mathbf{v}(\mathbf{x})=\mathbf{z} e^{\frac{1}{c} \sqrt{\sigma_{t} \mu} x}$ is solution to the $P_{N}$ model. Applying a rotation as in Proposition 2.1 gives the solutions (10). Moreover, considering $\lambda=-\sqrt{\sigma_{t} \mu} / c$ is equivalent to take $-\mathbf{d}_{k}$ in (10). We conclude that all the solutions (9) or (11) are given by (10). Finally, from Proposition 2.5 there exists $m_{e}$ distinct pairs $\left(\mu_{i}, \mathbf{w}_{i}\right)$ solution of the eigenproblem associated with the matrix $\left(A A^{T}\right)^{-1} R_{1}$. This completes the proof.

Examples of such exponential solutions are constructed in Section 4 for the $P_{1}$ and the $P_{3}$ models .

\subsection{Secular equation}

Exponential solutions require the eigenvalues and eigenvectors of the matrix $\left(A A^{T}\right)^{-1} R_{1}$. If $\sigma_{s}=0$, then $R_{1}=\varepsilon \sigma_{a} I_{m_{e}}$ is a diagonal matrix: then $\left(A A^{T}\right)^{-1} R_{1}$ is a symmetric matrix. But on the contrary, the matrix $\left(A A^{T}\right)^{-1} R_{1}$ is non symmetric in the general case $\sigma_{s} \neq 0$, and so, it can be difficult to solve directly this eigenvalue problem (see however some formulas in [16, Lemma 2]). Here we describe an alternative method based on the solution of a secular equation $[2,28]$. The key observation is that the departure of $R_{1}$ to diagonal matrices is a rank one matrix.

Technically, in our case, one can construct the secular equation making the distinction between eigenvectors which have a zero first component and eigenvectors which have a non zero first component. First case: eigenvectors with a zero first component. Take $\mathbf{w}_{i}$ an eigenvector of $\left(A A^{T}\right)^{-1}$ associated with the eigenvalue $\lambda_{i}$ and assume that its first component of $\mathbf{w}_{i}$ is null. In this case $R_{1} \mathbf{w}_{i}=\sigma_{t} \mathbf{w}_{i}$. So $\mathbf{w}_{i}$ is also an eigenvector of $\left(A A^{T}\right)^{-1} R_{1}$ associated with the eigenvalue $\lambda=\sigma_{t} \lambda_{i}$.

Second case: eigenvectors with a non zero first component. Now take $\mathbf{w}$ an eigenvector of the matrix $\left(A A^{T}\right)^{-1} R_{1}$ associated with the eigenvalue $\lambda$ and make the assumption that its first component of $\mathbf{w}$ is non zero. One starts from $R_{1} \mathbf{w}=A A^{T} \lambda \mathbf{w}$ : diagonalizing the matrix $A A^{T}=P D P^{T}$ $\left(P^{T} P=I_{m_{e}}\right)$ and using $R_{1}:=I_{m_{e}} \sigma_{t}-\frac{\sigma_{s}}{\varepsilon} \mathbf{e}_{1} \mathbf{e}_{1}^{T}, \mathbf{e}_{1}=(1,0, \ldots, 0)^{T} \in \mathbb{R}^{m_{e}}$, one gets

$$
\lambda D \widetilde{\mathbf{w}}=\left(I_{m_{e}} \sigma_{t}-\frac{\sigma_{s}}{\varepsilon} \mathbf{v} \mathbf{v}^{T}\right) \widetilde{\mathbf{w}},
$$

where $\widetilde{\mathbf{w}}=P^{T} \mathbf{w}$ and $\mathbf{v}=P^{T} \mathbf{e}_{1}$ is the vector of the first component of all eigenvectors of the matrix $A A^{T}$. The key is that the matrix $\mathbf{v v}^{T}$ in (15) can be seen as a rank one perturbation of diagonal matrices. One has $\left(\sigma_{t} I_{m_{e}}-\lambda D\right) \widetilde{\mathbf{w}}=\frac{\sigma_{s}}{\varepsilon} \mathbf{v} \mathbf{v}^{T} \widetilde{\mathbf{w}}$, so

$$
\widetilde{\mathbf{w}}=\left(I_{m_{e}} \sigma_{t}-\lambda D\right)^{-1} \frac{\sigma_{s}}{\varepsilon} \mathbf{v} \mathbf{v}^{T} \widetilde{\mathbf{w}} .
$$

Multiplying by $\mathbf{v}^{T}$ on both sides give

$$
\mathbf{v}^{T} \widetilde{\mathbf{w}}=\left(\mathbf{v}^{T}\left(I_{m_{e}} \sigma_{t}-\lambda D\right)^{-1} \frac{\sigma_{s}}{\varepsilon} \mathbf{v}\right) \mathbf{v}^{T} \widetilde{\mathbf{w}}
$$

Because $\mathbf{v}^{T}=\mathbf{e}_{1}^{T} P$ and $\widetilde{\mathbf{w}}=P^{T} \mathbf{w}$ one has $\mathbf{v}^{T} \widetilde{\mathbf{w}}=\mathbf{e}_{1}^{T} \mathbf{w}$. By assumption the first component of $\mathbf{w}$ is non zero, that is $\mathbf{v}^{T} \widetilde{\mathbf{w}} \neq 0$. One can simplify $\mathbf{v}^{T} \widetilde{\mathbf{w}}$ on both sides of the equality. It yields a scalar equation

$$
1=\frac{\sigma_{s}}{\varepsilon} \mathbf{v}^{T}\left(I_{m_{e}} \sigma_{t}-\lambda D\right)^{-1} \mathbf{v} .
$$


Setting $f(\lambda)=1+\frac{\sigma_{s}}{\varepsilon} \mathbf{v}^{T}\left(\lambda D-I_{m_{e}} \sigma_{t}\right)^{-1} \mathbf{v}$, one gets what is called the secular equation

$$
f(\lambda)=0 .
$$

Denoting $D=\operatorname{diag}\left(d_{1}>0, \ldots, d_{m_{e}}>0\right)$, the function $f$ can be written

$$
f(\lambda)=1+\frac{\sigma_{s}}{\varepsilon} \sum_{i=1}^{m_{e}} \frac{v_{i}^{2}}{\lambda d_{i}-\sigma_{t}} .
$$

Lemma 3.2. The function $f$ has a pole at $\sigma_{t} / d_{i}$ if and only if $v_{i} \neq 0$. It is a monotone function. For each pole, $f$ admits a root on the left side of the pole.

Proof. It is sufficient to compute the derivative $f^{\prime}(\lambda)=-\frac{\sigma_{s}}{\varepsilon} \sum_{i} d_{i} \frac{v_{i}^{2}}{\left(\lambda d_{i}-\sigma_{t}\right)^{2}}<0$ and to notice that $f(-\infty)=1$.

From (16) and denoting $C=\mathbf{v}^{T} \widetilde{\mathbf{w}} \neq 0$ one has $\widetilde{\mathbf{w}}=C\left(I_{m_{e}} \sigma_{t}-\lambda D\right)^{-1} \frac{\sigma_{s}}{\varepsilon} \mathbf{v}$. Therefore, once one has the eigenvalue $\lambda$, one deduces the associated eigenvector $\mathbf{w}$.

Example 3.3 ( $P_{1}$ model). It is a follow up of Example 2.1. One has $A A^{T}=\frac{1}{3}$. The eigenvalue is $d_{1}=\frac{1}{3}$ associated with the eigenvector $\mathbf{w}_{1}=1$. The function $f$ reads

$$
f(\lambda)=1+\frac{\sigma_{s}}{\varepsilon\left(\lambda d_{1}-\sigma_{t}\right)} .
$$

This function admits one roots $\lambda_{1}=3 \varepsilon \sigma_{a}$ which is also the only eigenvalue of the scalar matrix $\left(A A^{T}\right)^{-1} R_{1}=3 \varepsilon \sigma_{a}$.

Example 3.4 ( $P_{3}$ model). An illustration of the secular equation is provided in Figure 2 for the $P_{3}$ model. One can verify on (23) that two vectors have a zero first component and two vectors have a non zero first component. It yields two poles and two roots.

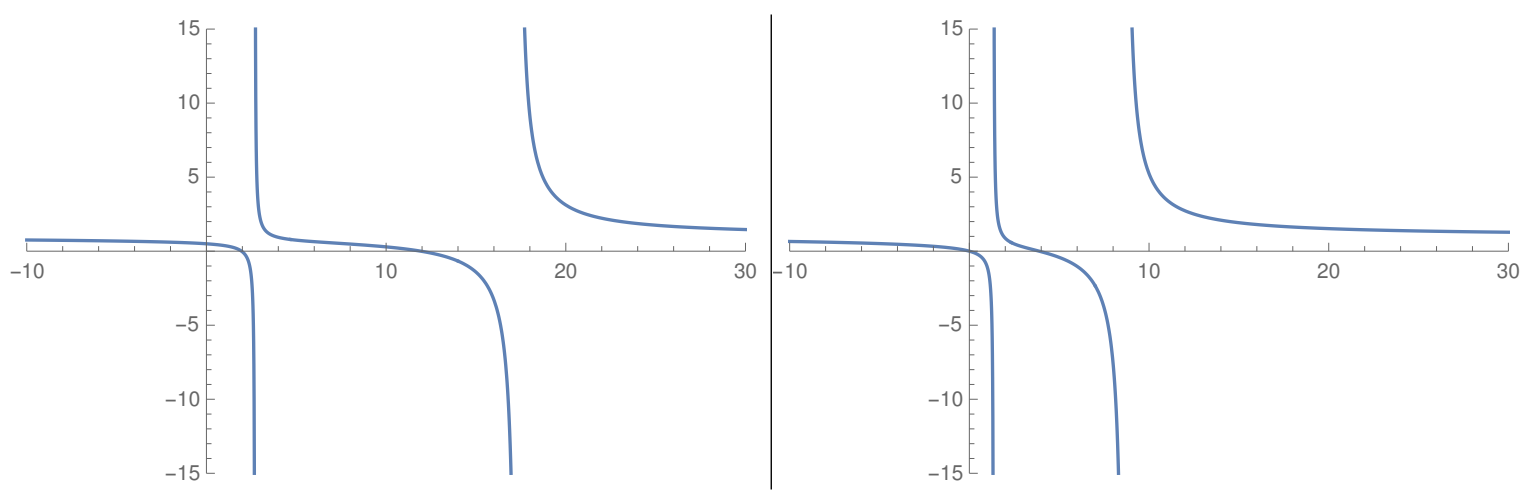

Figure 2: Representation of the function $f$ for the $P_{3}$ model. On the left $\sigma_{a}=\sigma_{s}=1$, on the right $\sigma_{a}=0, \sigma_{s}=1$.

\subsection{Harmonic polynomial solutions with Birkhoff and Abu-Shumays work}

So far we have constructed solutions with an exponential structure (9), we call them exponential solutions. If all eigenvalues $\mu_{i}$ are non zero in the representation formula (10), the intuition tells that such functions are linear independent for different directions $\mathbf{d}_{k}$. However if $\sigma_{a}=0$, then the situation is very different since the $\mu_{1}=0$ (Proposition 2.4) so the exponential factor degenerates 
(equal to 1). It may result in linear dependent functions. Unfortunately this is exactly what shows up, and this is a important issue in view of implementation because linearly dependent basis functions yield singular matrices after discretization. With this regard the situation is the same as the one described in [17] for plane wave basis of the Helmholtz equation when the frequency tends to zero: in the cited reference, the authors show that convenient rescaling of the exponential functions yield special polynomial functions. In our case, we describe an abstract method, based on the Birkhoff and Abu-Shumays approach, which constructs polynomial solutions for $\sigma_{a}=0$. Another method in nature closer to the Gittelson, Hiptmair and Perugia method [17] can be found in [30]. Hereafter, we present the general principles of the Birkhoff and Abu-Shumays work on the transport equation with a particular symmetry hypothesis which simplifies a lot the proof. A general proof with tools coming from the theory of potential is in the cited reference, together with an extension to the case $\sigma_{a}>0$ using hypergeometric functions. This is complemented by an original result in Theorem 3.7 which shows that projection of the kinetic function onto harmonic polynomial preserves the structure.

Take $q(x, y)$ which is harmonic polynomial $\Delta q(x, y)=0$. The series of all harmonic polynomials can be generated as follows: firstly set $q_{1}(x, y)=1$, then consider the series for $l \geq 1$

$$
q_{2 k}(\mathbf{x})=\frac{1}{k !} \Re\left(\left(x-x_{0}\right)+i\left(y-y_{0}\right)\right)^{k} \text { and } q_{k l+1}(\mathbf{x})=\frac{1}{k !} \Im\left(\left(x-x_{0}\right)+i\left(y-y_{0}\right)\right)^{k}
$$

Define the function which depends on a given harmonic polynomial $q$

$$
f(x, y, \boldsymbol{\Omega}):=\sum_{k=0}^{\infty}\left(\frac{-1}{\sigma_{s}}\right)^{k}(\boldsymbol{\Omega} \cdot \nabla)^{k} q(x, y), \quad l \geq 0,
$$

where $\boldsymbol{\Omega}:=(\sin \phi \cos \psi, \sin \phi \sin \psi, \cos \phi)^{T} \in \mathbb{R}^{3}$ with $\psi \in[0,2 \pi)$ and $\phi \in[0, \pi)$. Actually the series is finite and the function $f$ is a polynomial with respect to $x$ and $y$ : its degree is $\operatorname{deg}(q)$ which is the degree of the polynomial $q$. The terms of the series can be evaluated with the following formula.

Lemma 3.5. One has

$$
(\boldsymbol{\Omega} \cdot \nabla)^{k} q(x, y)=\left(\frac{\sin \phi}{2}\right)^{k}\left(e^{-i k \psi}\left(\partial_{x}+i \partial_{y}\right)^{k}+e^{i k \psi}\left(\partial_{x}-i \partial_{y}\right)^{k}\right) q(x, y), \quad k \geq 1 .
$$

Proof. Indeed since $\partial_{z} q=0$ one has

$$
(\boldsymbol{\Omega} \cdot \nabla) q(x, y)=\sin \phi\left(\cos \psi \partial_{x}+\sin \psi \partial_{y}\right) q(x, y)=\frac{\sin \phi}{2}\left(e^{i \psi}\left(\partial_{x}-i \partial_{y}\right)+e^{-i \psi}\left(\partial_{x}+i \partial_{y}\right)\right) q(x, y) .
$$

Therefore

$$
\begin{aligned}
(\boldsymbol{\Omega} \cdot \nabla)^{k} q(x, y) & =\left(\frac{\sin \phi}{2}\right)^{k} \sum_{p=0}^{k} C_{k}^{p} e^{i p \psi}\left(\partial_{x}-i \partial_{y}\right)^{p} e^{-i(k-p) \psi}\left(\partial_{x}+i \partial_{y}\right)^{k-p} q(x, y), \\
& =\left(\frac{\sin \phi}{2}\right)^{k} \sum_{p=0}^{k} C_{k}^{p} e^{i(2 p-k) \psi}\left(\partial_{x}-i \partial_{y}\right)^{p}\left(\partial_{x}+i \partial_{y}\right)^{k-p} q(x, y) .
\end{aligned}
$$

But since $q(x, y)$ is harmonic one has $\left(\partial_{x}-i \partial_{y}\right)\left(\partial_{x}+i \partial_{y}\right) q(x, y)=0$. Therefore, all the terms in the sum vanish except the first and the last. One finally finds the claim.

Proposition 3.6 ([4]). For $\sigma_{a}=0$, the function $f(x, y, \boldsymbol{\Omega})$ is solution to the stationary transport equation (4).

Proof. By definition one has $(\boldsymbol{\Omega} \cdot \nabla) f(x, y, \boldsymbol{\Omega})=\sum_{k=0}^{\infty}\left(\frac{-1}{\sigma_{s}}\right)^{k}(\boldsymbol{\Omega} \cdot \nabla)^{k+1} q(x, y)$. That is

$$
(\boldsymbol{\Omega} \cdot \nabla) f^{l}(x, y, \boldsymbol{\Omega})=\sigma_{s} q(x, y)-\sigma_{s} \sum_{k=0}^{\infty}\left(\frac{-1}{\sigma_{s}}\right)^{k}(\boldsymbol{\Omega} \cdot \nabla)^{k} q(x, y) .
$$

Using Lemma 3.5, one gets $q=|f|$. 
Our main result below explains the interest of the previous result for $P_{N}$ models. We note $\Pi_{N}$ the orthogonal projection onto the space of harmonic functions of degree less than $m=\frac{1}{2}(N+1)(N+2)$.

Theorem 3.7. Assume $\sigma_{a}=0$. Take $m=\frac{1}{2}(N+1)(N+2) \geq \operatorname{deg}(q)$. Then the function

$$
\mathbf{f}_{N}(x, y)=<\mathbf{y}(\boldsymbol{\Omega}) \Pi_{N} f(x, y, \boldsymbol{\Omega})>\in \mathbb{R}^{m^{3 D}},
$$

is solution to the $\Pi_{N}$ model and is harmonic polynomial with respect to $x, y$, with degree $\operatorname{deg}(q)$.

Proof. Let $\mathbf{y}(\boldsymbol{\Omega})$ be the collection of spherical harmonics (see for example [22, Appendix A]) up to order $m=\frac{1}{2}(N+1)(N+2)$. Since $m \geq \operatorname{deg}(q)$, the function $\Pi_{N} f(x, y, \boldsymbol{\Omega})$ can be decomposed on the spherical harmonics of degree less than $m$, namely $\Pi_{N} f(x, y, \boldsymbol{\Omega})=\mathbf{y}^{T}(\boldsymbol{\Omega}) \mathbf{f}_{N}(x, y)$. One starts from

$$
\langle\mathbf{y}(\boldsymbol{\Omega})(\boldsymbol{\Omega} \cdot \nabla) f(x, y, \boldsymbol{\Omega})\rangle=\sigma_{s}(-\langle\mathbf{y}(\boldsymbol{\Omega}) f(x, y, \boldsymbol{\Omega})\rangle+\langle\mathbf{y}(\boldsymbol{\Omega})\rangle\langle f(x, y, \boldsymbol{\Omega})\rangle) .
$$

It yields

$$
\begin{gathered}
\left\langle\mathbf{y}(\boldsymbol{\Omega})(\boldsymbol{\Omega} \cdot \nabla) \mathbf{y}^{T}(\boldsymbol{\Omega}) \mathbf{f}_{N}(x, y)\right\rangle+\left\langle\mathbf{y}(\boldsymbol{\Omega})(\boldsymbol{\Omega} \cdot \nabla) \sum_{k \geq m+1}\left(\frac{-1}{\sigma_{s}}\right)^{k}(\boldsymbol{\Omega} \cdot \nabla)^{k} q(x, y)\right\rangle \\
=\sigma_{s}\left(-\left\langle\mathbf{y}(\boldsymbol{\Omega}) \mathbf{y}^{T}(\boldsymbol{\Omega}) \mathbf{f}_{N}(x, y)\right\rangle+\langle\mathbf{y}(\boldsymbol{\Omega})\rangle\left\langle\mathbf{y}^{T}(\boldsymbol{\Omega}) \mathbf{f}_{N}(x, y)\right\rangle\right) .
\end{gathered}
$$

Using (19), the second term can be decomposed as an sum of terms proportional to $\left\langle\mathbf{y}(\boldsymbol{\Omega})(\sin \phi)^{k} e^{ \pm i k \psi}\right\rangle$. Now $\mathbf{y}(\boldsymbol{\Omega})$ is made of harmonic polynomials of maximal degree less than $m$ while $e^{ \pm i k \psi}$ is an exponential with degree $\geq m+2$ : so the integral with respect to $\psi$ make the product to vanish. The remaining terms are rewritten as

$$
\sum_{i=1}^{3}\left\langle\Omega_{i} \mathbf{y}(\boldsymbol{\Omega}) \mathbf{y}^{T}(\boldsymbol{\Omega})\right\rangle \partial_{x_{i}} \mathbf{f}_{N}(x, y)=\sigma_{s}\left(-\left\langle\mathbf{y}(\boldsymbol{\Omega}) \mathbf{y}^{T}(\boldsymbol{\Omega})\right\rangle+\langle\mathbf{y}(\boldsymbol{\Omega})\rangle\left\langle\mathbf{y}^{T}(\boldsymbol{\Omega})\right\rangle\right) \mathbf{f}_{N}(x, y) .
$$

Using the orthonormality $\left\langle\mathbf{y y}^{T}\right\rangle=I_{m}$, one recognizes the stationary version of the full 3D $P_{N}$ model for $\sigma_{a}=0$, where $A_{i}=\left\langle\Omega_{i} \mathbf{y}(\boldsymbol{\Omega}) \mathbf{y}^{T}(\boldsymbol{\Omega})\right\rangle$.

We refer to $[16,30]$ for additional details. By retaining the components of $f_{N}$ which satisfy additional invariance principles (mainly points c) and d) in Section 2), one gets polynomial solutions to model (1).

\section{Application to $P_{1}$ and $P_{3}$}

In this section, we give explicitly the stationary solutions to the $P_{1}$ and $P_{3}$ models.

\subsection{The $P_{1}$ model}

We recall that the matrices read

$$
A=\left(\begin{array}{cc}
\frac{1}{\sqrt{3}} & 0
\end{array}\right), \quad B=\left(\begin{array}{cc}
0 & \frac{1}{\sqrt{3}}
\end{array}\right), \quad R_{1}=\varepsilon \sigma_{a}, \quad R_{2}=\left(\begin{array}{cc}
\sigma_{t} & 0 \\
0 & \sigma_{t}
\end{array}\right) .
$$

We are interested in the stationary solutions to the $P_{1}$ model. Such solutions have already been calculated in [31]. We start with the exponential solutions when $\sigma_{a}>0$.

Proposition 4.1. Take $\mathbf{d}_{k}=\left(\cos \theta_{k}, \sin \theta_{k}\right)^{T} \in \mathbb{R}^{2}$. The following functions are solution to the $P_{1}$ model

$$
\mathbf{v}_{k}=\left(\begin{array}{c}
\sqrt{\sigma_{t}} \\
-\sqrt{\varepsilon \sigma_{a}} \mathbf{d}_{k}
\end{array}\right) e^{\frac{1}{c} \sqrt{3 \varepsilon \sigma_{a} \sigma_{t}} \mathbf{d}_{k}^{T} \mathbf{x}}, \quad \sigma_{t}=\varepsilon \sigma_{a}+\frac{\sigma_{s}}{\varepsilon} .
$$


Proof. We use the Theorem 3.1. From the definition of the matrices $A$ and $R_{1}$ (20) associated with the $P_{1}$ model, one has $\left(A A^{T}\right)^{-1} R_{1}=3 \varepsilon \sigma_{a}$. This matrix has one eigenvalue $\mu_{1}=3 \varepsilon \sigma_{a}$ associated with the eigenvector $\mathbf{w}_{1}=1$. Taking the notations from Theorem 3.1, one has $\mathbf{z}_{1}=\left(1,-\sqrt{\frac{\varepsilon \sigma_{a}}{\sigma_{t}}}, 0\right)^{T}$. Using the definition of the rotation matrix $U_{\theta_{k}}$ (see Example 2.1 or [30, Section 4.1.3.2]) and multiplying the solution by $\sqrt{\sigma_{t}}$ give the functions (21). The proof is complete.

Now, we describe all polynomial solutions when $\sigma_{a}=0$.

Proposition 4.2. Take $\sigma_{a}=0$. Let $q$ be any harmonic polynomial. The following function is a solution to the $P_{1}$ model

$$
\mathbf{v}_{k}=\left(\begin{array}{c}
\frac{\sigma_{s}}{\varepsilon} q \\
-\frac{c}{\sqrt{3}} \nabla q
\end{array}\right)
$$

Proof. It can be deduced from the Birkhoff and Abu-Shumays method. However a direct proof is immediate. Using the definition (20) of the matrices $A, B$ and $R$ associated with the $P_{1}$ model one gets $\frac{c}{\sqrt{3}} \partial_{x} v_{1}=-\sigma_{t} v_{2}$ and $\frac{c}{\sqrt{3}} \partial_{y} v_{1}=-\sigma_{t} v_{3}$. Using $\sigma_{t}=\frac{\sigma_{s}}{\varepsilon}$ completes the proof.

One notices that a direct verification that harmonic polynomial of any order are solutions to $P_{1}$ for $\sigma_{a}=0$. This is a consequence of Theorem 3.7.

\subsection{The $P_{3}$ model}

First, we calculate the stationary exponential solutions.

Proposition 4.3. Take $\mathbf{d}_{k}=\left(\cos \theta_{k}, \sin \theta_{k}\right)^{T} \in \mathbb{R}^{2}$. The following functions are solutions to the $P_{3}$ model

$$
\begin{aligned}
& \mathbf{v}_{1}(\mathbf{x})=\left(\begin{array}{c}
0 \\
-\sqrt{30} \cos 2 \theta_{k} \\
0 \\
\sqrt{30} \sin 2 \theta_{k} \\
\sqrt{14} \cos \theta_{k} \\
-\sqrt{14} \sin \theta_{k} \\
\sqrt{15} \cos 3 \theta_{k} \\
-\cos \theta_{k} \\
\sin \theta_{k} \\
-\sqrt{15} \sin 3 \theta_{k}
\end{array}\right) e^{\frac{1}{c} \sqrt{\frac{7}{3}} \sigma_{t} \mathbf{d}_{k}^{T} \mathbf{x}}, \quad \mathbf{v}_{2}(\mathbf{x})=\left(\begin{array}{c}
0 \\
\sqrt{2} \sin 2 \theta_{k} \\
\sqrt{6} \\
\sqrt{2} \cos 2 \theta_{k} \\
0 \\
0 \\
-\sqrt{3} \sin 3 \theta_{k} \\
-\sqrt{5} \sin \theta_{k} \\
-\sqrt{5} \cos \theta_{k} \\
-\sqrt{3} \cos 3 \theta_{k}
\end{array}\right) e^{\frac{1}{c} \sqrt{7} \sigma_{t} \mathbf{d}_{k}^{T} \mathbf{x}}, \\
& \mathbf{v}_{3}(\mathbf{x})=\left(\begin{array}{c}
\frac{\sqrt{\sigma_{t}}}{14 \sqrt{15}} \rho^{+} \\
\varepsilon \sqrt{\sigma_{t}} \sigma_{a} \sin 2 \theta_{k} \\
\frac{\varepsilon \sqrt{\sigma_{t}} \sigma_{a}}{\sqrt{3}} \\
\varepsilon \sqrt{\sigma_{t}} \sigma_{a} \cos 2 \theta_{k} \\
-\frac{1}{630 \sqrt{2}} v^{-} \tau^{+} \sin \theta_{k} \\
-\frac{1}{630 \sqrt{2}} v^{-} \tau^{+} \cos \theta_{k} \\
-\frac{\varepsilon}{2 \sqrt{21}} \sigma_{a} v^{-} \sin 3 \theta_{k} \\
\frac{\varepsilon}{2 \sqrt{35}} \sigma_{a} v^{-} \sin \theta_{k} \\
\frac{\varepsilon}{2 \sqrt{35}} \sigma_{a} v^{-} \cos \theta_{k} \\
-\frac{\varepsilon}{2 \sqrt{21}} \sigma_{a} v^{-} \cos 3 \theta_{k}
\end{array}\right) e^{\frac{1}{c} v^{-} \sqrt{\frac{\sigma t}{18}} \mathbf{d}_{k}^{T} \mathbf{x}}, \quad \mathbf{v}_{4}(\mathbf{x})=\left(\begin{array}{c}
\frac{\sqrt{\sigma_{t}}}{14 \sqrt{15}} \rho^{-} \\
\varepsilon \sqrt{\sigma_{t}} \sigma_{a} \sin 2 \theta_{k} \\
\frac{\varepsilon \sqrt{\sigma_{t}} \sigma_{a}}{\sqrt{3}} \\
\sqrt{\sigma_{t}} \sigma_{a} \cos 2 \theta_{k} \\
-\frac{\sqrt{\varepsilon}}{630 \sqrt{2}} v^{+} \tau^{-} \sin \theta_{k} \\
-\frac{\sqrt{\varepsilon}}{630 \sqrt{2}} v^{+} \tau^{-} \cos \theta_{k} \\
-\frac{\sqrt{\varepsilon}}{2 \sqrt{21}} \sigma_{a} v^{+} \sin 3 \theta_{k} \\
\frac{\sqrt{\varepsilon}}{2 \sqrt{35}} \sigma_{a} v^{+} \sin \theta_{k} \\
\frac{\sqrt{\varepsilon}}{2 \sqrt{35}} \sigma_{a} v^{+} \cos \theta_{k} \\
-\frac{\sqrt{\varepsilon}}{2 \sqrt{21}} \sigma_{a} v^{+} \cos 3 \theta_{k}
\end{array}\right) e^{\frac{1}{c} v^{+} \sqrt{\frac{\sigma_{t}}{18}} \mathbf{d}_{k}^{T} \mathbf{x}},
\end{aligned}
$$

with $\sigma_{t}=\varepsilon \sigma_{a}+\frac{\sigma_{s}}{\varepsilon}$ and where we use the following notations $\kappa=\sqrt{605 \varepsilon^{2} \sigma_{a}^{2}+14 \varepsilon \sigma_{a} \sigma_{t}+245 \sigma_{t}^{2}}$, $v^{ \pm}=\sqrt{55 \varepsilon \sigma_{a}+35 \sigma_{t} \pm \sqrt{5} \kappa}, \tau^{ \pm}=\sqrt{5} \varepsilon \sigma_{a}+35 \sqrt{5} \sigma_{t} \pm 5 \kappa, \rho^{ \pm}=\left(v^{ \pm}\right)^{2}-110 \varepsilon \sigma_{a}$. 
Proof. With the definitions (8) of the matrices $A$ and $R_{1}$, one finds that the matrix $\left(A A^{T}\right)^{-1} R_{1}$ admits the following eigenvalues $\mu_{1}=\frac{7}{3} \sigma_{t}, \mu_{2}=7 \sigma_{t}, \mu_{3}=\frac{\left(v^{-}\right)^{2}}{18}$ and $\mu_{4}=\frac{\left(v^{+}\right)^{2}}{18}$, and the following eigenvectors

$$
\mathbf{w}_{1}=\left(\begin{array}{l}
0 \\
1 \\
0 \\
0
\end{array}\right), \quad \mathbf{w}_{2}=\left(\begin{array}{c}
0 \\
0 \\
\sqrt{3} \\
1
\end{array}\right), \quad \mathbf{w}_{3}=\left(\begin{array}{c}
\frac{-11 \sqrt{5} \varepsilon \sigma_{a}+7 \sqrt{5} \sigma_{t}+\kappa}{14 \sqrt{3} \varepsilon} \\
0 \\
-\frac{\sigma_{a}}{\sqrt{3}} \\
\sigma_{a}
\end{array}\right), \quad \mathbf{w}_{4}=\left(\begin{array}{c}
\frac{-11 \sqrt{5} \varepsilon \sigma_{a}-7 \sqrt{5} \sigma_{t}+\kappa}{14 \sqrt{3} \varepsilon} \\
0 \\
-\frac{\sigma_{a}}{\sqrt{3}} \\
\sigma_{a}
\end{array}\right) .
$$

See, for example, [30, Section 4.1.3.2]. After easy simplifications, and considering the correct scaling, one finds the functions (23).

Remark 4.4. For $\sigma_{a}=0$, then $v^{-}=\mu_{3}=0$ and the exponential functions associated to the third family degenerate. See also the Figure 2. This phenomenon is essentially the same one observed in [17] for the Helmholtz equation in the low frequency regime.

Proposition 4.5. Take $\sigma_{a}=0$. The following polynomial functions are solutions

$$
\begin{gathered}
\mathbf{v}_{1}(\mathbf{x})=\left(\begin{array}{l}
1 \\
0 \\
0 \\
0 \\
0 \\
0 \\
0 \\
0 \\
0 \\
0
\end{array}\right), \quad \mathbf{v}_{2}(\mathbf{x})=\left(\begin{array}{c}
\sigma_{t} x \\
0 \\
0 \\
0 \\
0 \\
-\frac{c}{\sqrt{3}} \\
0 \\
0 \\
0 \\
0
\end{array}\right), \quad \mathbf{v}_{3}(\mathbf{x})=\left(\begin{array}{c}
\sigma_{t} y \\
0 \\
0 \\
0 \\
-\frac{c}{\sqrt{3}} \\
0 \\
0 \\
0 \\
0 \\
0
\end{array}\right), \\
\mathbf{v}_{4}(\mathbf{x})=\left(\begin{array}{c}
\sigma_{t}^{2} x y \\
\frac{2 c^{2}}{\sqrt{15}} \\
0 \\
0 \\
-\frac{\sigma_{t} c}{\sqrt{3}} x \\
-\frac{\sigma_{t} c}{\sqrt{3}} y \\
0 \\
0 \\
0 \\
0 \\
0
\end{array}\right), \quad \mathbf{v}_{5}(\mathbf{x})=\left(\begin{array}{c}
\frac{1}{2} \sigma_{t}^{2}\left(x^{2}-y^{2}\right) \\
0 \\
0 \\
\frac{2 c^{2}}{\sqrt{15}} \\
\frac{\sigma_{t} c}{\sqrt{3}} y \\
-\frac{\sigma_{t} c}{\sqrt{3}} x \\
0 \\
0 \\
0 \\
0 \\
0
\end{array}\right)
\end{gathered}
$$

Proof. Three different proofs are possible.

- The first one is by far the simplest one: it relies on injecting the formulas (24) into the Friedrichs system (1) and to verify they are exact.

- The second one is to consider the Birkhoff and Abu-Shumays method with $q=1, x, y, x y$ and $x^{2}-y^{2}$ and to perform additional calculations to determine the components of the vectorial function $f_{3}$ in Theorem 3.7.

- A third fully constructive method which is however heavier is in Morel's PhD thesis [30].

\section{$5 \quad$ Numerical illustration}

Various $h$-convergence results (theoretical and numerical) are available in the literature for TDG methods for time harmonic equations $[11,15,17]$. For time harmonic wave equation, $p$-convergence is ana- 
lyzed in [23]. For the family of Friedrichs systems evaluated in this work, $h$-convergence can be found in $[29,31,30]$.

The validation of the new basis functions that is proposed below is based more on the notion of adaptivity, which is different than $h$ or $p$-convergence. Adaptivity is perhaps a more relevant concept for the industrial use of numerical methods. In what follows we consider a coarse mesh, and compare the results obtained with TDG with results obtained with a more traditional polynomial based DG method.

\subsection{A test problem with a boundary layer}

In this test, taken from [31], a two dimensional test with discontinuous coefficients is studied. The numerical method is a Discontinuous Galerkin method with the Trefftz exact solutions computed so far. The structure of the numerical code is classical: assemble matrix and right hand side, invert matrix, display results. It is described in [30].

The domain is $\Omega=[0,1]^{2}$ and we define $\Omega_{1}$ (resp. $\Omega_{2}$ ) as $\Omega_{1}=[0.35,0.65]^{2}$ (resp. $\Omega_{2}=\Omega \backslash \Omega_{1}$ ). We take $\varepsilon=1, c=1$ and

$$
\sigma_{a}=2 \times \mathbf{1}_{\Omega_{1}}(\mathbf{x}), \quad \sigma_{s}=2 \times \mathbf{1}_{\Omega_{2}}(\mathbf{x})+10^{5} \times \mathbf{1}_{\Omega_{1}}(\mathbf{x}) .
$$

The absorption coefficient has compact support in $\Omega_{1}$ while the scattering coefficient is discontinuous and takes a high value in $\Omega_{1}$. Referring to the initial model (1), these coefficients involve a truly discontinuous matrix $R$. Even if we consider random meshes, the interface between $\Omega_{1}$ and $\Omega_{2}$ is a straight line. The geometry and parameters of this test are represented in Figure 3.

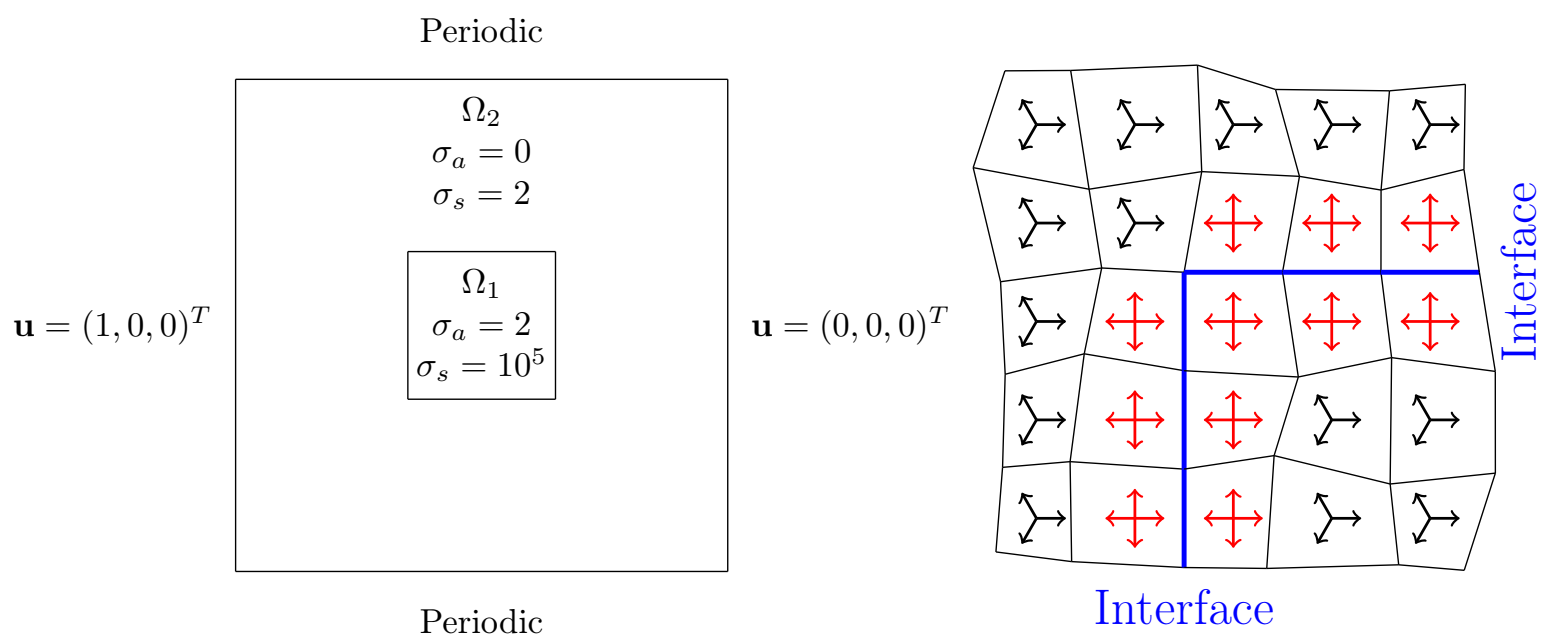

Figure 3: On the left: Domain and boundary condition for the two dimensional boundary layers test. On the right: representation of adaptive directions at the interface. In this example: the 3 equidistributed directions (25) in each cell except at the interface where the directions are locally adapted into (26).

This test has already been performed for the two dimensional $P_{1}$ model [31]. We focus here on the $P_{3}$ model. For the directions, we consider three possible choices. With 3 basis functions per cell, we may consider the following equi-distributed directions

$$
\mathbf{d}_{1}=(1,0)^{T}, \quad \mathbf{d}_{2}=\left(\cos \frac{2 \pi}{3}, \sin \frac{2 \pi}{3}\right)^{T}, \quad \mathbf{d}_{3}=\left(\cos \frac{4 \pi}{3}, \sin \frac{4 \pi}{3}\right)^{T} .
$$

With 4 basis functions per cell, we may consider the following equi-distributed directions

$$
\mathbf{d}_{1}=(1,0)^{T}, \quad \mathbf{d}_{2}=(0,1)^{T}, \quad \mathbf{d}_{3}=(-1,0)^{T}, \quad \mathbf{d}_{4}=(0,-1)^{T} .
$$


With 5 basis functions per cell, we may consider the following equi-distributed directions

$$
\begin{gathered}
\mathbf{d}_{1}=(1,0)^{T}, \quad \mathbf{d}_{2}=\left(\cos \frac{2 \pi}{5}, \sin \frac{2 \pi}{5}\right)^{T}, \quad \mathbf{d}_{3}=\left(\cos \frac{4 \pi}{5}, \sin \frac{4 \pi}{5}\right)^{T}, \\
\mathbf{d}_{4}=\left(\cos \frac{6 \pi}{5}, \sin \frac{6 \pi}{5}\right)^{T}, \quad \mathbf{d}_{5}=\left(\cos \frac{8 \pi}{5}, \sin \frac{8 \pi}{5}\right)^{T} .
\end{gathered}
$$

As pointed in [31], the choice of directions at the interface plays an important role to correctly capture the boundary layers. In particular, it seems essential to locally get the one dimensional direction perpendicular to the interface associated with the boundary layer. Therefore, we make the special choice of directions (26) at the interface. Such directions are well adapted if one considers the one dimensional problem at the interface. A graphical illustration of the adaptive directions at the interface is provided on the right of Figure 3. As stated previously, when $\sigma_{a}=0$ the degenerative exponentials are replaced with polynomials. With our parameters, the number of polynomials used in the basis functions is equal to the number of directions.
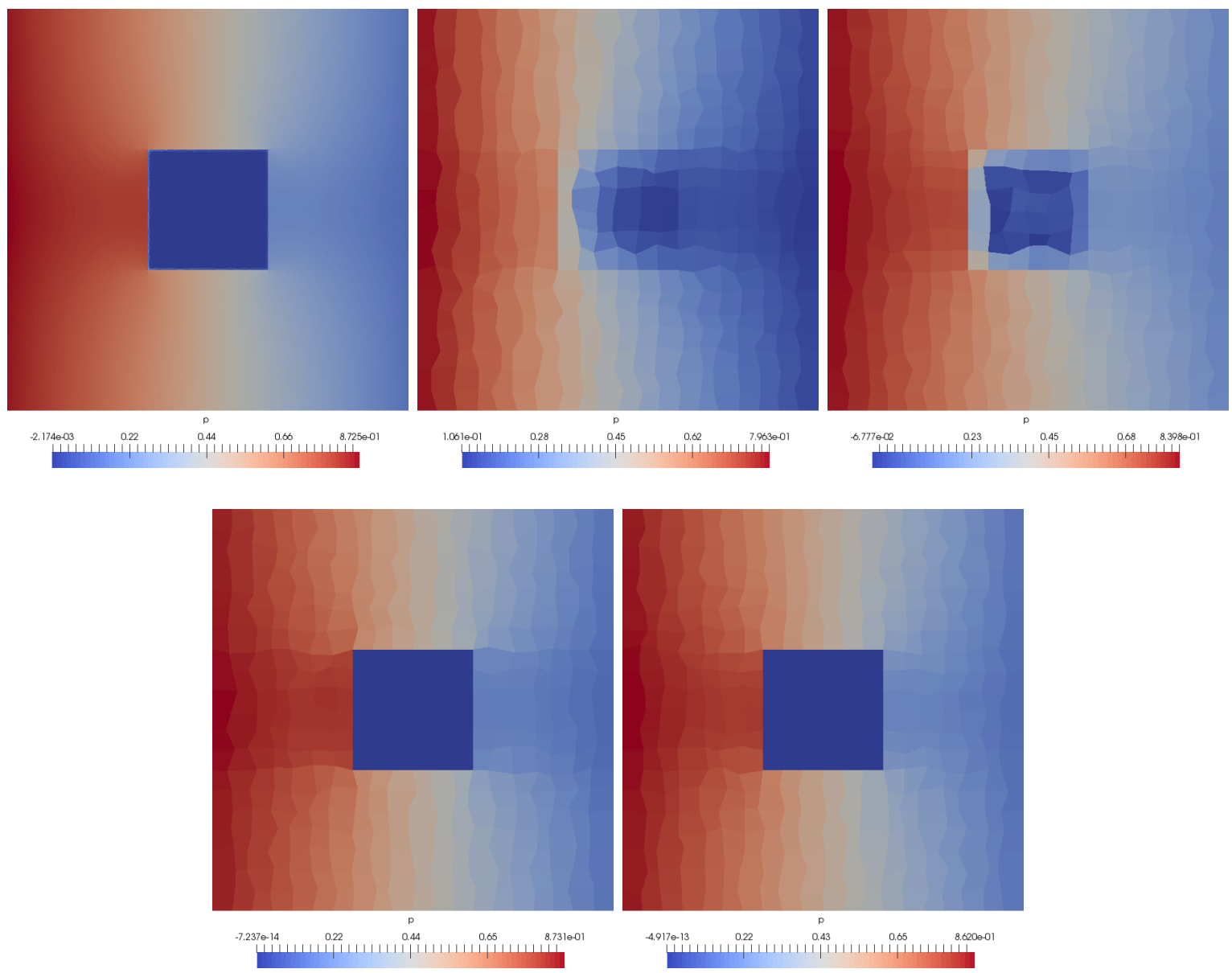

Figure 4: Representation of the first variable for the $P_{3}$ model. Top left: reference solution. Top center: DG scheme with 10 basis functions per cell. Top right: DG scheme with 30 basis functions per cell. Bottom left: TDG scheme with 12 basis functions per cell. Bottom right: TDG scheme with 20 basis functions per cell. For the TDG scheme, the directions at the interface are locally adapted into the 4 directions (26).

The reference solution is calculated on a $200 \times 200$ random mesh with the 3 directions (25) and 
adaptive directions (26) at the interface. We compare the following cases on a coarse $20 \times 20$ mesh

- The DG method with constant basis functions only (= finite volume) for a total of 10 basis functions per cell.

- The DG method with affine basis functions (that is $1, x, y$ ) for a total of 30 basis functions per cell.

- The TDG method with the basis functions (23)-(24) depending on the 3 directions (25), for a total of 12 basis functions per cell, and on the 4 directions (26) at the interface.

- The TDG method with the basis functions (23)-(24) depending on the 5 directions (27), for a total of 20 basis functions per cell, and the 4 directions (26) at the interface.

The results given in Figure 4 are very similar to the $P_{1}$ case tested in [31]. One notices a better approximation of the solution for the TDG method with less degrees of freedom compared to the standard DG scheme.

If the TDG method gives such good result, it is in fact because the correct exponential solutions (i.e. with the right directions) are locally used in the boundary layers. Actually, an enrichment strategy, where the DG basis is locally (in the boundary layers) enriched with some exponential solutions, would give similar result on this numerical test [30, Section 5-4.3.2]. The same kind of idea is used, for example, in the context of the so-called extended finite element method (XFEM) [1, 14].

\subsection{A test problem in the regime $\sigma_{a}=0$}

For the $P_{3}$ model we compare the results obtained with the DG and TDG method on a $80 \times 80$ mesh. The time step is $\Delta t=T / 80$. We consider four different simulations:

- The limit solution which is the fundamental solution of the $2 \mathrm{D}$ heat equation.

- The DG method with constant basis functions only (= finite volume) for a total of 10 basis functions per cell.

- The DG method with affine basis function (that is $1, x$ and $y$ ) for a total of 30 basis functions per cell.

- The TDG method initially with the basis made of three directions for the exponential functions. But the degenerate exponential function (that is $\mathbf{v}_{3}$ in (23)) is systematically removed. Instead it is replaced by the first three polynomial functions $\mathbf{v}_{1,2,3}$ in (24) That is the TDG method applied to the $P_{3}$ model uses a combination of exponential and polynomial basis functions. For an implementation with five directions, one replaces the degenerate exponentials with $\mathbf{v}_{1,2,3,4,5}$.

The results presented in Figure 5 illustrate that the DG method with only constant basis functions is too diffusive. On the contrary, one recovers a good approximation with the TDG method. This illustrates the good behavior of TDG approximations for such problems. The DG scheme with affine basis functions is also accurate, but with the disadvantage of using approximately three times more basis functions than the TDG scheme.

\section{Conclusion}

We have presented various ways to construct exact solutions to a family of Friedrichs systems with relaxation, which in this study comes from an angular approximation of the transport equation with linear relaxation. Two families of exact stationary solutions were proposed. The first one contains exponential factors and is valid for $\sigma_{a}>0$. The second one contains polynomial terms and is valid for $\sigma_{a}=0$. In practice, for $\sigma_{a}=0$, one may use a combination of both. A general procedure to 

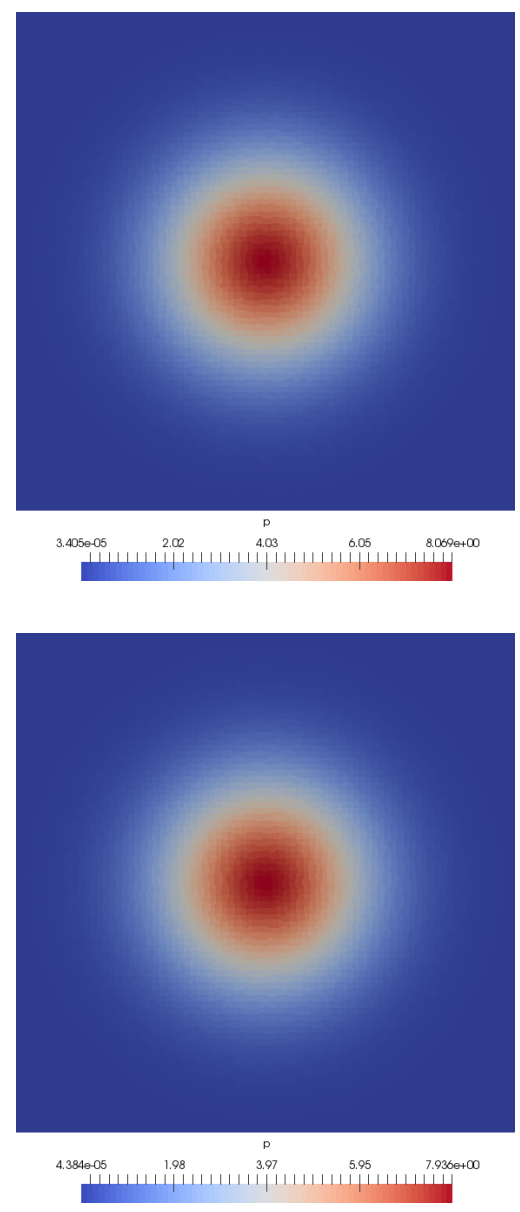
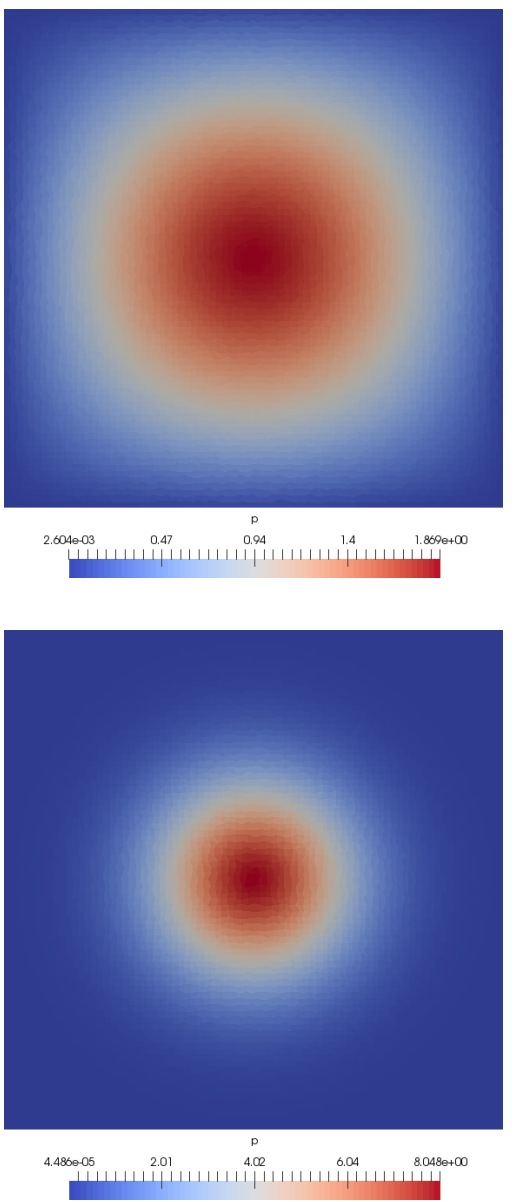

Figure 5: Top left: limit solution. Top right: DG- $P_{0}=\mathrm{FV}$ solution with 10 basis functions per cell. Bottom left: DG- $P_{1}$ solution with 30 basis functions per cell. Bottom right: TDG solution with 12 basis functions per cell.

construct basis functions with time dependence is sketched in the appendix. All these exact functions can be generalized in $3 \mathrm{D}$. The $S_{N}$ can be treated with a similar approach, except that the invariance by rotation is discrete for equi-distributed velocity directions, not continuous as for the $P_{N}$ model.

The validation proposed in this work is based on the comparaison of the results of the classical DG code with the one of a TDG code. Actually it is the same code, only the new basis functions have been introduced and the matrix and right hand side recomputed accordingly. For the results presented, the TDG method shows improved accuracy with respect to the traditional DG method.

A comprehensive numerical analysis of the better accuracy of TDG for the problems discussed is probably beyond what is permitted by the available mathematical theory so far. It should be the subject of further research.

\section{A Time dependent solutions}

We give some possible ways to get time dependent solutions to the $P_{N}$ model. These solutions can also be used as basis functions for the TDG method when considering space-time mesh. Many other time dependent solutions can be constructed starting from [10, 5]. 
- A first possibility is to consider one dimensional solution under the form

$$
\mathbf{v}(t, x)=\mathbf{q}(t, x) e^{\lambda x},
$$

where $\mathbf{q}(t, x) \in \mathbb{R}^{m}$ is polynomial vector in $x$ ant $t$. A concrete example is given in [31, Proposition 4.2] for the case of the $P_{1}$ model. Then one can use the rotational invariance of the $P_{N}$ model and gets the following solutions

$$
\mathbf{v}(t, \mathbf{x})=U_{\theta} \mathbf{q}(t, x \cos \theta+y \sin \theta) e^{\lambda(x \cos \theta+y \sin \theta)} .
$$

Another possibility is to search directly for two dimensional solutions under the form

$$
\mathbf{v}(t, \mathbf{x})=\mathbf{p}(t, \mathbf{x}) e^{\lambda(x \cos \theta+y \sin \theta)},
$$

where $\mathbf{p}(t, \mathbf{x}) \in \mathbb{R}^{m}$ is polynomial vector in $x, y$ ant $t$. Note that the functions obtained with (29) may differ from the functions (28). A complete example is given in [30, Section 5.2.2] for the $P_{1}$ model.

- Another possibility is to consider time dependent solutions under the form

$$
\mathbf{v}(t, \mathbf{x})=\mathbf{g}(\mathbf{x}) e^{\alpha t},
$$

with $\alpha \in \mathbb{R}$. One can inject this solution in the $P_{N}$ model (1). One gets after removing the exponentials

$$
\left(A_{1} \partial_{x}+A_{2} \partial_{y}+\left(R+\varepsilon \alpha I_{m}\right)\right) \mathbf{g}(\mathbf{x})=\mathbf{0},
$$

where $I_{m}$ is the identity matrix of $\mathbb{R}^{m \times m}$. The function $\mathbf{g}(\mathbf{x})$ is very similar to the stationary solutions already calculated in Section 3.1. The matrix $R$ is just replaced by the matrix $\widetilde{R}:=$ $R+\varepsilon \alpha I_{m}$.

For example, if one takes $\alpha$ such that $\sigma_{a}+\varepsilon \alpha>0$, then $\mathbf{g}(\mathbf{x})$ is one of the exponential solutions (10). In particular, if $\alpha>0$, the functions (30) naturally degenerate toward non trivial time dependent solutions when $\sigma_{a} \rightarrow 0$. This is one asset of the functions (30).

\section{References}

[1] Y. Abdelaziz and A. Hamouine, A survey of the extended finite element, Computers \& Structures, 86 (2008), pp. 1141 - 1151, doi:https://doi.org/10.1016/j.compstruc.2007.11.001, http://www.sciencedirect.com/science/article/pii/S0045794907002957.

[2] J. Anderson, A secular equation for the eigenvalues of a diagonal matrix perturbation, Linear Algebra and its Applications, 246 (1996), pp. 49 - 70, doi:http://dx.doi.org/10.1016/00243795(94)00314-9.

[3] C. Berthon and R. Turpault, Asymptotic preserving HLL schemes., Numer. Methods Partial Differ. Equations, 27 (2011), pp. 1396-1422, doi:10.1002/num.20586.

[4] G. Birkhoff and I. Abu-Shumays, Harmonic solutions of transport equations., J. Math. Anal. Appl., 28 (1969), pp. 211-221, doi:10.1016/0022-247X(69)90123-1.

[5] G. Birkhoff and I. Abu-Shumays, Exact analytic solutions of transport equations., J. Math. Anal. Appl., 32 (1970), pp. 468-481, doi:10.1016/0022-247X(70)90271-4.

[6] A. M. Blanco, M. Florez, and M. Bermejo, Evaluation of the rotation matrices in the basis of real spherical harmonics, 419 (1997), pp. 19-27. 
[7] T. A. Brunner And J. P. Holloway, One-dimensional Riemann solvers and the maximum entropy closure, Journal of Quantitative Spectroscopy and Radiative Transfer, 69 (2001), pp. 543 - 566, doi:https://doi.org/10.1016/S0022-4073(00)00099-6, http://www . sciencedirect. com/science/article/pii/S0022407300000996.

[8] C. Buet, B. Després, And E. Franck, Asymptotic preserving schemes on distorted meshes for Friedrichs systems with stiff relaxation: application to angular models in linear transport., J. Sci. Comput., 62 (2015), pp. 371-398.

[9] A. Buffa And P. Monk, Error estimates for the Ultra Weak Variational Formulation of the Helmholtz equation, ESAIM: Mathematical Modelling and Numerical Analysis, 42 (2008), pp. 925940 .

[10] K. CASE, Elementary solutions of the transport equation and their applications., Ann. Phys., 9 (1960), pp. 1-23, doi:10.1016/0003-4916(60)90060-9.

[11] O. Cessenat and B. Després, Application of an Ultra Weak Variational Formulation of Elliptic PDEs to the Two-Dimensional Helmholtz Problem, SIAM J. Numer. Anal., 35 (1998), pp. 255-299.

[12] F. Dai And Y. Xu, Approximation theory and harmonic analysis on spheres and balls, New York, NY: Springer, 2013, doi:10.1007/978-1-4614-6660-4.

[13] B. Després And C. Buet, The structure of well-balanced schemes for Friedrichs systems with linear relaxation, Applied Mathematics and Computation, 272 (2016), pp. 440-459.

[14] T.-P. Fries And T. Belytschko, The extended/generalized finite element method: An overview of the method and its applications, International Journal for Numerical Methods in Engineering, 84 (2010), pp. 253-304, doi:10.1002/nme.2914, http://dx.doi.org/10.1002/nme.2914.

[15] G. GABARD, Exact integration of polynomial-exponential products with application to wavebased numerical methods, Comm. Numer. Methods Engrg., 25 (2009), pp. 237-246, doi:10.1002/cnm.1123, https://doi.org/10.1002/cnm.1123.

[16] C. Garrett And C. D. Hauck, On the eigenstructure of spherical harmonic equations for radiative transport., Comput. Math. Appl., 72 (2016), pp. 264-270, doi:10.1016/j.camwa.2015.05.030.

[17] C. J. Gittelson, R. Hiptmair, and I. Perugia, Plane wave discontinuous Galerkin methods: Analysis of the h-version., ESAIM, Math. Model. Numer. Anal., 43 (2009), pp. 297-331.

[18] J. Gopalakrishnan, P. Monk, and P. Sepúlveda, A tent pitching scheme motivated by Friedrichs theory, Comput. Math. Appl., 70 (2015), pp. 1114-1135, doi:10.1016/j.camwa.2015.07.001, https://doi.org/10.1016/j. camwa.2015.07.001.

[19] L. Gosse, Computing qualitatively correct approximations of balance laws. Exponential-fit, wellbalanced and asymptotic-preserving., Milano: Springer, 2013, doi:10.1007/978-88-470-2892-0.

[20] L. Gosse And G. Toscani, An asymptotic-preserving well-balanced scheme for the hyperbolic heat equations., C. R., Math., Acad. Sci. Paris, 334 (2002), pp. 337-342.

[21] J. M. Greenberg And A. Y. Leroux, A well-balanced scheme for the numerical processing of source terms in hyperbolic equations, SIAM Journal on Numerical Analysis, 33 (1996), pp. 1-16, doi:10.1137/0733001, https://doi.org/10.1137/0733001, arXiv:https://doi.org/10.1137/0733001.

[22] F. Hermeline, A discretization of the multigroup $P_{N}$ radiative transfer equation on general meshes., J. Comput. Phys., 313 (2016), pp. 549-582. 
[23] R. Hiptmair, A. Moiola, and I. Perugia, Plane wave discontinuous Galerkin methods for the 2D Helmholtz equation: analysis of the p-version, SIAM J. Numer. Anal., 49 (2011), pp. 264-284.

[24] R. Hiptmair, A. Moiola, and I. Perugia, A survey of Trefftz methods for the Helmholtz equation, in Building bridges: connections and challenges in modern approaches to numerical partial differential equations, vol. 114 of Lect. Notes Comput. Sci. Eng., Springer, [Cham], 2016, pp. 237-278.

[25] J. Ivanic And K. Ruedenberg, Rotation matrices for real spherical harmonics. direct determination by recursion, Journal of Physical Chemistry, 100 (1996), doi:10.1021/jp953350u.

[26] S. JIN, A steady-state capturing method for hyperbolic systems with geometrical source terms, in Transport in Transition Regimes, N. B. Abdallah, I. M. Gamba, C. Ringhofer, A. Arnold, R. T. Glassey, P. Degond, and C. D. Levermore, eds., New York, NY, 2004, Springer New York, pp. 177-183.

[27] S. Jin And C. Levermore, Numerical schemes for hyperbolic conservation laws with stiff relaxation terms., J. Comput. Phys., 126 (1996), pp. 449-467, art. no. 0149.

[28] S. Jin And D. LeVermore, The discrete-ordinate method in diffusive regimes, 20 (1991), pp. 413439.

[29] F. Kretzschmar, A. Moiola, I. Perugia, and S. M. Schnepp, A priori error analysis of space-time Trefftz discontinuous Galerkin methods for wave problems, IMA Journal of Numerical Analysis, 36 (2016), p. 1599.

[30] G. Morel, Asymptotic-preserving and well-balanced schemes for transport models using Trefftz discontinuous Galerkin method, PhD thesis, Sorbonne Université, 2018, https://hal. archives-ouvertes.fr/tel-01911872.

[31] G. Morel, C. Buet, And B. Després, Trefftz discontinuous Galerkin method for Friedrichs systems with linear relaxation: application to the $P_{1}$ model, Computational Methods in Applied Mathematics, (2018), pp. 521-557.

[32] D. Pinchon and P. E. Hoggan, Rotation matrices for real spherical harmonics: general rotations of atomic orbitals in space-fixed axes., J. Phys. A, Math. Theor., 40 (2007), pp. 1597-1610, doi:10.1088/1751-8113/40/7/011.

[33] I. K. Tezaur and C. Farhat, A discontinuous enrichment method for the finite element solution of high Péclet advection-diffusion problems, Finite Elements in Analysis and Design, (2009), pp. 238-250.

[34] O. Zienkiewicz, Origins, milestones and directions of the finite element method? a personal view, Archives of Computational Methods in Engineering, (1995), pp. 1-48. 